\title{
A METHOD FOR TREATING DISCRETIZATION ERROR IN NONDETERMINISTIC ANALYSIS
}

\author{
Kenneth F. Alvin ${ }^{*}$ \\ Structural Dynamics and Vibration Control Dept. \\ Sandia National Laboratories, Albuquerque, NM
}

\begin{abstract}
A response surface methodology-based technique is presented for treating discretization error in nondeterministic analysis. The response surface, or metamodel, is estimated from computer experiments which vary both uncertain physical parameters and the fidelity of the computational mesh. The resultant metamodel is then used to propagate the variabilities in the continuous input parameters, while the mesh size is taken to zero, its asymptotic limit. With respect to mesh size, the metamodel is equivalent to Richardson extrapolation, in which solutions on coarser and finer meshes are used to estimate discretization error. The method is demonstrated on a one dimensional prismatic bar, in which uncertainty in the third vibration frequency is estimated by propagating variations in material modulus, density, and bar length. The results demonstrate the efficiency of the method for combining nondeterministic analysis with error estimation to obtain estimates of total simulation uncertainty. The results also show the relative sensitivity of failure estimates to solution bias errors in a reliability analysis, particularly when the physical variability of the system is low.
\end{abstract}

* Senior Member Technical Staff, Structural Dynamics and Vibration Control Dept., P.O. Box 5800, MS 0439, Albuquerque, NM 87185-0439; Senior Member AIAA

Copyright $\odot 1999$ by the American Institute of Aeronautics and Astronautics, Inc. All rights reserved.
I. Introduction

Nondeterministic analysis methods are applied to simulations of physical systems in order to quantify the effects of random variations in system parameters and inputs on the predicted output of the simulation. Typically, nondeterministic methods are used to propagate probability or frequency distributions of continuous "physical" variables through a deterministic mapping, such as the discretized numerical solution of a system of partial differential equations (PDEs), plus boundary and initial conditions and auxiliary submodels. In this case, it is important to have a verified and validated model structure through which to propagate these continuous variabilities. However, all finite discretized models possess some degree of discretization error, and often little or no attempt is made to estimate the magnitude of discretization error in the model. Furthermore, even when some error estimate is available, it is unclear how to apply that estimate to the ensemble of results computed during a nondeterministic analysis. Thus, the effect of discretization error in nondeterministic analysis is practically never treated at the present time.

There has, however, been a significant amount of attention devoted to the problem of estimating errors in numerical methods for solving deterministic partial differential equations. Among these methods are a posteriori error estimators ${ }^{1,2}$, as well as such classical methods as Richardson extrapolation ${ }^{3-5}$. Of particular interest in this study is Richardson extrapolation, in which discretization errors are estimated from the numerical solution. Richardson extrapolation is extremely general in that it can be applied to any output of the model, as well as 


\section{DISCLAIMER}

This report was prepared as an account of work sponsored by an agency of the United States Government. Neither the United States Government nor any agency thereof, nor any of their employees, make any warranty, express or implied, or assumes any legal liability or responsibility for the accuracy, completeness, or usefulness of any information, apparatus, product, or process disclosed, or represents that its use would not infringe privately owned rights. Reference herein to any specific commercial product, process, or service by trade name, trademark, manufacturer, or otherwise does not necessarily constitute or imply its endorsement, recommendation, or favoring by the United States Government or any agency thereof. The views and opinions of authors expressed herein do not necessarily state or reflect those of the United States Government or any agency thereof. 


\section{DISCLAIMER}

Portions of this document may be illegible in electronic image products. Images are produced from the best available original document. 
functionals of the solution. Its primary drawback is that it depends on knowledge of the formal convergence rate of the numerical method and requires that the mesh size used is fine enough that the higher-order terms of the error are negligible compared to the lowest-order term. For this reason, it often requires more that two mesh spacings on the same model to verify the convergence order and thus establish the validity of the extrapolation.

A conservative approach to accounting for discretization error in a nondeterministic analysis (which relies on the numerical solution of PDEs) would be to use $\mathrm{Ri}$ chardson extrapolation (or some other error estimator) for every combination of input values to the model. For example, we might perform a structural dynamics simulation in which the elastic modulus of some material in the design model has some inherent variability. Then, for each particular value of that parameter we could compute the response on two or more spatial discretizations and use these results in some to-be-determined way in our nondeterministic analysis. This approach would then increase the number of analyses to be performed by a factor of two to three. On the other hand, we might consider performing error estimation for only one particular value of the variable parameter (such as its mean value) and then apply that error estimate in a relative or absolute sense to the analyses performed for other values of the input. This approach would require only a modest increase in computational cost compared to the cost of the nondeterministic analysis itself, but cannot account for the dependence of the error estimate on the values of the parameters of the model. It should be noted that the investment in generating multiple discrete models with different mesh spacings might be much more significant than the cost of computing solutions on each of the meshes.

In the present study, an alternative approach is considered in which solutions are computed on different mesh sizes, but not for every parameter value in the nondeterministic analysis. Instead, both mesh size and parameter values are varied for the purpose of building a surrogate model, or metamodel, for interpolation. Once solutions are computed to build the metamodel, a regression is performed to obtain the coefficients of the metamodel. The metamodel can then be used in the nondeterministic analysis in place of the complex full-order model. For example, frequency distributions on input variables can be easily propagated through the metamodel via Monte Carlo analysis, since the cost of computing a response based on the metamodel is negligible. The treatment of discretization error is accomplished by extending traditional response surface methods (RSM) for determining metamodels to include mesh size as a variable input parameter. Then the nondeterministic analysis can be performed for a mesh size of zero, which conforms to a higher-order accurate solution of the governing PDEs.

In this study, it has been found that including mesh size in the metamodel can be an efficient way to minimize discretization error while performing nondeterministic analysis. For example, a traditional metamodel with 3 continuous input variables might require 13 evaluations of the complex simulation with different parameter values to determine the parameters of the metamodel. If we wished to minimize discretization error, we would perform those 13 evaluations on a fine mesh (i.e. with small element edge lengths). By extending that model to include element edge length as an input variable of the metamodel, we must now perform 25 evaluations of the complex simulation. However, only 6 of those evaluations are performed on the fine mesh model, while the other evaluations are performed on coarser meshes which require much less time to solve. Therefore, it is possible to determine the extended metamodel with less overall computational effort. Furthermore, the extended metamodel can be used to estimate the converged continuum solution, while the traditional metamodel determined strictly from evaluations of the fine mesh model still suffers some overall unquantified error due to the discretization.

The remainder of the study is organized as follows. First, the technique for using response surface methodology for nondeterministic analysis is presented. Then, this methodology is extended to include the dependence of the simulation on mesh size. Finally, results are presented for linear dynamics of a prismatic bar with 3 uncertain input parameters. The results demonstrate the importance of treating discretization error when estimating system reliability measures such as probability of failure. In the example, discretization error on the order of $1 \%$ of the response quantity results in a probability of failure estimate which is more than one order of magnitude smaller than the exact solution, an error of more than $90 \%$. Thus, the bias caused by small discretization errors results in a significant overprediction of the reliability. The technique developed herein reduced the error in probability of failure to below $5 \%$.

\section{Response Surface Methodology for Nondeterministic Analysis}

Montgomery ${ }^{6}$ notes that

Response surface methodology, or RSM, is a collection of mathematical and statistical techniques that are useful for the modeling and analysis of problems in which a response of interest is influenced by several variables and the objective is to optimize the response.... 
The process yield is a function of the levels..., say

$$
y=f\left(x_{1}, x_{2}\right)+e
$$

where $e$ represents the noise observed in the response $y$. If we denote the expected response by $E[y]=f\left(x_{1}, x_{2}\right)=h$, then the surface represented by

$$
h=f\left(x_{1}, x_{2}\right)
$$

is called the response surface.

In the present context, we will use RSM as a surrogate or metamodel for the complex physics model of interest, and we will estimate the parameters of the response surface by performing a limited number of analyses of the complex model. Our goal is to use the response surface to propagate uncertainties in the variables to determine a distribution of the response quantity. From this distribution we can make estimates of failure probability or other statistics of interest.

The use of RSM is logically coupled to Design of Experiments (DOE). In order to apply RSM we must determine a selection of input vectors for the complex simulation. Designing the input vectors for the suite of simulations to be run is the objective of experiment design. A logical goal of DOE is to minimize the variance of the error between the response surface and the discrete responses of the complex model. It is important, however, to also consider the frequency distributions of the input parameters, as well as the type of statistic to be computed from the distribution of the output. Finally, as noted by Sacks ${ }^{7}$ there are fundamental differences between physical experiments and computer experiments which influence the issue of experiment design.

In this study, we focus on a simple global surface model which includes second-order terms in the parameters. The model response is given by

$$
y=\beta_{0}+\sum_{i} \beta_{i} p_{i}+\sum_{i} \sum_{j} \beta_{i j} p_{i} p_{j}
$$

where $\beta_{0}, \beta_{i}, \beta_{i j}$ are parameters of the metamodel to be estimated from analyses performed on the complex model. It should be noted that this metamodel form is just one possibility; other functions could be considered as well as the finite element lattice sampling approach of Romero ${ }^{8}$.

To this standard metamodel form we will apply the well-known Box-Behnken experiment design?, which dictates a set of input vectors for which the parameters $p_{i}$ take on nominal, high, or low values. It is suggested that these levels be taken as the mean and the mean plus or minus one to two standard deviations, respectively. Furthermore, the number of simulation runs is somewhat greater than the number of parameters being estimated, so that a least-squares estimate for the metamodel parameters is obtained. The Box-Behnken designs for 3 and 4 input variables are given in Table 1 and Table 2, where $0,+1,-1$ represent the nominal, high and low values, respectively.

Other possible designs are Central composite and sub-optimal iterate selection algorithms such as Effective Independence ${ }^{10}$ and Subset selection ${ }^{11}$.

Once an experiment design has been determined, and the computer experiments are performed, the parameters $\beta_{i j}$ of the meta-model are estimated, typically using a least-squares method.

\section{Extension of RSM to include Mesh Size}

The typical model for spatial discretization error is given as

$$
y(h)=y_{\text {exact }}+\alpha h^{q}+O\left(h^{q+1}\right)
$$

where $h$ is the characteristic mesh spacing or element length, $y(h)$ is the numerical solution resulting from the mesh, $y_{\text {exact }}$ is the exact solution of the corresponding continuum model, $q$ is the formal order of the method, and $\alpha$ is some unknown factor. Assuming the validity of this error model and knowledge of method order $q$, we could easily construct a metamodel from a small number of simulation runs with different values of $h$. Since the only unknowns are $\alpha$ and $y_{\text {exact }}$, we require only two different mesh spacings. With three different mesh spacings, we could also confirm the method order $q$. This approach of estimating the terms of the error model using the results from different mesh spacings is in fact just the classical Richardson extrapolation method.

Thus, given the similarity between RSM and Richardson extrapolation, it is reasonable to combine the two methods into a larger metamodel form in order to treat discretization error within the context of nondeterministic analysis. The form of the extended metamodel is taken to be

$$
\begin{array}{r}
y(p, h)=\beta_{0}+\sum_{i} \beta_{i} p_{i}+\sum_{i} \sum_{j} \beta_{i j} p_{i} p_{j}+ \\
\alpha_{0} h^{q}+\sum_{i} \alpha_{i} p_{i} h^{q}
\end{array}
$$

which allows for the metamodel to account for the coupling between the constant and linear terms of the nominal metamodel and the discretization error, but neglects the terms of $O\left(p^{2} h^{q}\right)$ and above. 


\section{A. Experiment Design for Extended Metamodel}

For experiment design purposes, we will again use the Box-Behnken design, where the number of input variables is increased by one for input value $h$. This requires that we develop discretized models with 3 different mesh spacings. As with Richardson extrapolation, it is usually desirable to perform mesh doubling. The values $-1,0,+1$ with respect to $h$ are strictly qualitative and correspond to coarse, nominal and fine mesh models, respectively. For example, re-interpreting Table 2 for the case of 3 input parameters plus variation in mesh fidelity, we have the experiment design given in Table 3. The value $h / h_{n o m}$ implicitly assumes the use of mesh doubling, but this is not specifically required.

The efficiency of the present method can be seen in examining Tables 1 and 3 . When the metamodel is extended by one input variable in order to include the mesh size parameter $h$, the total number of analyses increases, but the number performed with a fine mesh is only a small fraction of the total and less than the total number of runs of the non-extended metamodel. Thus, a traditional response surface (without $h$ ) estimated by analyses performed using the fine mesh can actually be less efficient than extending the model to include $h$, since the total computational effort is dictated by the number of fine mesh analyses performed. This comparison is summarized in Table 4 for different numbers of continuous input variables. Note that as the number of input parameters increase, the efficiency gains become dramatic.

\section{B. Verification of Formal Order of Convergence}

In a real sense, the nominal mesh model, together with the coarse mesh model, is being used to infer or interpolate the effects of the parameter variations which would seen at the fine mesh level. The mechanism for performing this inference is the form of the response surface, which is grounded in the error model Eqn. (2). This error model is valid, however, only in the asymptotic range and thus imposes a restriction on the fidelity of the meshes considered. In a sobering demonstration of the limitations of Richardson's method, Oberkampf and Blottner ${ }^{12}$ showed that for a particular system of nonlinear equations with large local gradients, reaching the asymptotic range for Richardson's method required very fine grids with relative errors on the order of $0.1 \%$. Therefore, computational meshes which simply meet an analyst's subjective criteria for "goodness" cannot be immediately assumed to meet the asymptotic range requirements of Richardson's method and the present extended response surface technique. It is important to assess the convergence characteristics for the range of meshes con- sidered and demonstrate the formal convergence of the method before proceeding with extrapolative procedures.

There are two approaches to verifying the convergence order using the experiment design procedure discussed above. Examining Table 3 for the particular case of 3 metamodel input parameters, there are 6 points in the design space where solutions are computed using both the coarse and fine meshes of the model. Two mesh sizes are not sufficient, however, to independently estimate both the mesh error and the convergence order $q$. In order to independently estimate $q$ we also require a solution at these design points for the nominal mesh. These can be obtained by performing additional computer experiments using the nominal mesh at these design points (corresponding to extreme values of each parameter). Alternatively, we could use the results from the runs on the nominal mesh to build a traditional metamodel with respect to the input parameters conditioned on the nominal mesh, and then use that metamodel to interpolate the response for the nominal mesh at the design points where coarse and fine mesh runs have been performed.

\section{Nondeterministic Analysis with Extended Metamodel}

The final stage of the nondeterministic analysis involves the use of the metamodel to propagate the parametric uncertainties through to the simulation output. From the distribution of the output, probabilistic or statistical quantities such as probability of failure, expected output value and variance can be estimated. One straightforward approach is what Romero terms decoupled Monte Carlo (DMC) analysis. Decoupled refers to the fact that the building of the response surface from complex simulation runs and the Monte Carlo analysis using the response surface are separate activities. For the Monte Carlo analysis, we would generate a very large number of samples from the joint distribution function of the uncertain variables and compute the metamodel response for each sample to build the output distribution. The only modification required for the extended metamodel is determining how to sample the mesh spacing parameter $h$.

Given that we are attempting to approximate the continuum problem, a mesh size of $h=0$ should be used for all evaluations of the metamodel. The only caveat on this specification is that this is equivalent to extrapolation of the metamodel, a practice which is usually avoided in RSM. However, extrapolation is exactly what the Richardson method uses for discretization error estimation, and is why care must be taken in verifying the formal order of convergence. Hence, if a similar level of care is taken with the extended metamodel, it should be possible to use the mesh size $h=0$ with confidence. Fi- 
nally, if there is still concern with using the metamodel to extrapolate, it is possible to simply use the fine mesh size value $h_{\text {fine }}$ for all evaluations of the metamodel. This will minimize discretization error without extrapolation outside the boundaries of the complex model evaluations.

\section{Example: 1-D Prismatic Bar}

The first example is a uniform prismatic bar with fixed-free boundary conditions as shown in Figure 1. The objective of the analysis is to estimate the probability of failure of the bar, where failure is defined by the condition $f_{3}<16,000 \mathrm{~Hz}$, where $f_{3}$ is the frequency of the third mode of vibration. The physical parameters defining the system are given in Table 5, where the coefficient of variation is equal to the standard deviation of the distribution divided by the mean. The mesh chosen for the nominal model is also shown in Figure 1. Note that the mesh was chosen to be non-uniform. There are two reasons for this. First, in most complex problems it is difficult or impossible to achieve a uniform grid, and often the mesh is refined in some areas to enhance accuracy for some output of the model. Secondly, it is understood that numerical methods tend to behave better in a theoretical sense on uniform grids, thus the non-uniform grid in this problem is intended to make a very simple problem somewhat more difficult. From the fixed end to the free end, the element lengths are: 4 elements @ 1.25 in, 4 elements@1.00 in, 4 elements@ 0.75 in, 4 elements @ 0.50 in, 4 elements@ 0.25 in. Thus there is a total of 20 elements and 20 degrees of freedom (d.o.f.) in the nominal model. The coarse and fine meshes for this problem are related to the nominal mesh by doubling and halving the element lengths, respectively. Therefore, the coarse mesh has 10 d.o.f. and the fine mesh has 40 d.o.f.

The experiment design given in Table 3 was used for estimating the extended metamodel, with the interpolation function given by Eqn. (3) and $q=2$ as the formal order of the method. Then the decoupled Monte Carlo analysis was performed with 100,000 samples of the joint density function of $E, \rho$, and $L$. All samples were evaluated at $h=0$. For comparison, a direct Monte Carlo analysis was performed using the exact continuum solution ${ }^{13}$ :

$$
f_{3}=\frac{5 \sqrt{E / \rho}}{4 L}
$$

Also for comparison, two approximate solutions are treated. First, a direct Monte Carlo analysis was performed using the nominal mesh model. Second, a decoupled Monte Carlo analysis was performed for the fine mesh model, where the response surface was estimated with respect to the 3 physical variables $E, \rho$, and $L$ using Eqn. (1) and the experiment design given in Table 1. Finally, histograms of each of the 4 Monte Carlo analyses were computed using 1000 bins, and the results input to a kernel density estimator to arrive at estimates of the output probability density functions. These final results are shown in Figure 2.

\section{Discussion of Results}

The results from this example are summarized in Table 6. For each of the methods, the total number of finite element analyses are given, as well as the number of analyses using the fine resolution grid of discretization. Then the total number of computations required for the nondeterministic analysis are given in Mflop ( $10^{6}$ floating point operations). Finally, the error in the estimated mean of the distribution, as well as the estimated probability of failure, which is the statistic of interest in the problem.

Note first of all that the continuum solution is the exact result, subject to Monte Carlo sampling errors (which are small because of the number of samples). The rest of the methods are given down the table in order of their accuracy. Interestingly, the extended RSM method is not only the most accurate approximation, but is also the most efficient. The more traditional RSM based on the fine mesh is still a reasonable result, although it requires more computations and is somewhat less accurate than the extended RSM. Finally, the direct Monte Carlo method based on the nominal mesh is not only very inefficient, but also leads to a significant error in the probability of failure estimate.

Apart from the comparison of methods, one of the most interesting aspects of these results is the effect the prediction error, as measured by the error in the estimated mean of the distribution, has on the relative error in the probability of failure estimate. In the case of the direct Monte Carlo analysis based on the nominal mesh, the discretization error results in a prediction error of about $1 \%$ of the exact solution. This would normally be considered more than adequate for engineering purposes. However, this error leads to a probability of failure estimate which is over one order of magnitude smaller than the exact solution. Thus, the reliability of the system might be judged to be more than 10 times higher than its actual reliability. The heightened sensitivity is a function of the probability of failure magnitude we are trying to estimate, as well as the coefficient of variation (the ratio of the standard deviation of the distribution to the mean value) of the simulation output used in the failure calculation. 
This can be seen in Figures 3 and 4, which show the relative error in the estimated probability of failure as a function of the failure threshold and as a function of the true failure probability. The jumps in the curves at the left hand side of the figures is due to finite sample errors in the "exact" solution. From Figure 3, it can be seen that as the failure threshold is increased beyond $16 \mathrm{kHz}$, all of the failure probability estimates increase towards 1.0, and the error relative to the exact failure probability reduces gradually. When these relative errors are plotted as a function of the true failure probability as in Figure 4, the problem appears even worse. Unfortunately, when performing reliability analyses on high consequence systems, we are typically computing very small magnitude failure estimates. This analysis not only confirms the hypothesis that far higher computational accuracy is required for modeling and simulation based life cycle engineering (MSBLCE) than for traditional engineering analysis, but also suggests that even the most conservative estimates of accuracy requirements for MSBLCE may be inadequate for reliability computations. However, the methodology presented herein appears to be a reasonable approach to treating and correcting discretization errors in the context of nondeterministic analyses.

\section{Summary and Conclusions}

A technique has been presented for treating discretization error in nondeterministic analysis. The technique involves the use of response surface methodology (RSM), in which a metamodel representation of a complex simulation model is estimated from a limited number of computer experiments on the complex model. The metamodel is then extended to include the characteristic element edge length as an model input parameter. The extended metamodel is used to propagate the variabilities in the continuous input parameters, while the mesh size is taken to zero, its asymptotic limit. The technique is demonstrated on a one dimensional prismatic bar, in which the uncertainty in the frequency of the third mode of vibration is estimated by propagating variations in the elastic modulus and mass density of the material, together with variation in the total length of the bar. Results are compared to the closed-form solution, a direct Monte Carlo analysis using a nominal mesh size, and a traditional response surface without a mesh size parameter built from computer experiments using a fine mesh size. The results demonstrate the importance of treating discretization error when estimating system reliability measures such as probability of failure, and the efficiency of the present technique for combining nondeterministic analysis with error estimation to obtain more accurate estimates of total simulation uncertainty.

\section{Acknowledgments}

The author would like Bill Oberkampf and Brian Rutherford at Sandia, as well as Dan Kammer of UWMadison, for contributing to this work through many discussions, as well as reviewing its content. Any errors and omissions, however, are the sole responsibility of the author. The support of the Sandia Computer Sciences LDRD program is also gratefully acknowledged. Sandia is a multiprogram laboratory operated by Sandia Corporation, a Lockheed Martin Company, for the United States Department of Energy under Contract DE-AC0494AL85000.

\section{REFERENCES}

1. Zienkiewwicz, O. C., and Zhu, J. Z., "A Simple Error Estimator and Adaptive Procedure for Practical Engineering Analysis," Int. J. Numer. Methods Eng., 24, 337-357 (1987).

2. Babuska, I., Strouboulis, T., Upadhyay, C. S., Gangaraj, S. K., and Copps, K., "Validation of $A$ Posteriori Error Estimators by Numerical Approach," Int. J. Numer. Methods Eng., 37, 1073-1123 (1994).

3. Richardson, L. F., "The Approximate Arithmetical Solution by Finite Differences of Physical Problems Involving Differential Equations with an Application to the Stresses in a Masonry Dam," Trans. $R$. Soc., Lond., Ser. A, 210 (1910).

4. Richards, S. A., "Completed Richardson Extrapolation in Space and Time," Comm. Numer. Methods Eng., 13, 573-582 (1997).

5. Westerink, J. J., and Roache, P. J., "Issues in Convergence Studies in Geophysical Flow Computations," ASME Fluids Engineering Div. Pub. FEDVol. 213, Quantification of Uncertainty in Computational Fluid Dynamics, ASME, Aug. 1995.

6. Montgomery, D. C., Design and Analysis of Experiments, 3rd Ed., John Wiley \& Sons, New York, 1991.

7. Sacks, J., Welch, W. J., Mitchell, T. J., and Wynn, H. P., "Design and Analysis of Computer Experiments," Statistical Science, Vol. 4, No. 4, pp. 409435 (1989).

8. memo, Sandia National Labs., from V. J. Romero and S. D. Bankston, 9113, to D. R. Martinez, 9234, subject: Application of decoupled Monte Carlo analysis with Finite Element / Lattice Sampling response surface for multimodal test problem (Function 1), dated July 7, 1997. 
9. Fox, E. P., "Issues in Utilizing Response Surface Methodologies for Accurate Probabilistic Design," Paper AIAA-96-1496-CP, AIAA, Washington DC (1996).

10. Kammer, D. C., "Sensor Placement for On-Orbit Modal Identification and Correlation of Large Space Structures," Journal of Guidance, Dynamics and Controls, 14(2), pp. 251-259 (1991).

11. Red-Horse, J. R., "Modal Experiment Design Using Subset Selection," Proceedings of the 14th Int. Modal Analysis Conf., Detroit, MI, Feb. 1996.

12. Oberkampf, W. L., and Blottner, F. G., "Issues in Computational Fluid Dynamics Code Verification and Validation," AIAA Journal, Vol. 36, No. 5, 1998, pp. 687-695.

13. Weaver, W., Timoshenko, S. P., Young, D. H., Vibration Problems in Engineering, 5th Ed., John Wiley \& Sons, New York, 1990, pp. 369-370.

Table 1: Box-Behnken Design for 3 Input Variables

\begin{tabular}{rrrr} 
Runs & $\mathrm{p}(1)$ & $\mathrm{p}(2)$ & $\mathrm{p}(3)$ \\
\hline $1-4$ & $+/-1$ & $+/-1$ & 0 \\
$5-8$ & 0 & $+/-1$ & $+/-1$ \\
$9-12$ & $+/-1$ & 0 & $+/-1$ \\
13 & 0 & 0 & 0 \\
\hline \hline
\end{tabular}

Table 2: Box-Behnken Design for 4 Input Variables

\begin{tabular}{rrrrr} 
Runs & $\mathrm{p}(1)$ & $\mathrm{p}(2)$ & $\mathrm{p}(3)$ & $\mathrm{p}(4)$ \\
\hline $1-4$ & $+/-1$ & $+/-1$ & 0 & 0 \\
$5-8$ & 0 & $+/-1$ & $+/-1$ & 0 \\
$9-12$ & 0 & 0 & $+/-1$ & $+/-1$ \\
$13-16$ & $+/-1$ & 0 & $+/-1$ & 0 \\
$17-20$ & 0 & $+/-1$ & 0 & $+/-1$ \\
$21-24$ & $+/-1$ & 0 & 0 & $+/-1$ \\
25 & 0 & 0 & 0 & 0 \\
\hline \hline
\end{tabular}

Table 3: Box-Behnken Design for 3 Input Variables plus Mesh Fidelity Variation

\begin{tabular}{rrrrrr} 
Runs & $\mathrm{p}(1)$ & $\mathrm{p}(2)$ & $\mathrm{p}(3)$ & Mesh & $\frac{h}{h_{n o m}}$ \\
\hline $1-4$ & $+/-1$ & $+/-1$ & 0 & nominal & 1.0 \\
$5-8$ & 0 & $+/-1$ & $+/-1$ & nominal & 1.0 \\
$9-12$ & 0 & 0 & $+/-1$ & coarse & 2.0 \\
$11-12$ & 0 & 0 & $+/-1$ & fine & 0.5 \\
$13-16$ & $+/-1$ & 0 & $+/-1$ & nominal & 1.0 \\
$17-18$ & 0 & $+/-1$ & 0 & coarse & 2.0 \\
$19-20$ & 0 & $+/-1$ & 0 & fine & 0.5 \\
$21-22$ & $+/-1$ & 0 & 0 & coarse & 2.0 \\
$23-24$ & $+/-1$ & 0 & 0 & fine & 0.5 \\
25 & 0 & 0 & 0 & nominal & 1.0 \\
\hline \hline
\end{tabular}

Table 4: Efficiency Gains by using Extended Metamodel with Mesh parameter

Number of Metamodel inputs (not including $h$ )

Efficiency ${ }^{\mathrm{a}}$

\begin{tabular}{rr}
\hline 3 & 2.2 \\
4 & 3.1 \\
5 & 6.7 \\
6 & 8.3 \\
\hline
\end{tabular}

a.

$\left[\frac{\# \text { fine mesh runs in extended model }}{\text { total \# runs in non-extended model }}\right]^{-1}$ 
Table 5: Parameter Information for 1-D Bar

\begin{tabular}{rlrrr}
\hline Parameter & Distribution & Mean Value & $\begin{array}{r}\text { Coefficient of } \\
\text { Variation }\end{array}$ \\
\hline \hline Elastic & $\mathrm{E}$ & Normal & $\begin{array}{r}10 \times 10^{6} \\
\mathrm{psi}\end{array}$ & $1.0 \%$ \\
Modulus & & & $\begin{array}{r}0.000259 \\
\mathrm{lb}-\mathrm{s}^{2} / \mathrm{in}^{2}\end{array}$ & $1.0 \%$ \\
Mass & $\rho$ & Normal & \\
Density & & & 15.0 in & $1.0 \%$ \\
Total & $\mathrm{L}$ & Normal & & \\
Length & & & &
\end{tabular}

Table 6: Nondeterministic Analysis Results for 1-D Bar Example

\begin{tabular}{|c|c|c|c|c|c|}
\hline Method & $\begin{array}{r}\text { Total \# } \\
\text { FEM } \\
\text { Analyses }\end{array}$ & $\begin{array}{r}\text { \# Fine Grid } \\
\text { FEM } \\
\text { Analyses }\end{array}$ & $\begin{array}{r}\text { \# Computations } \\
\text { (MFlop) }\end{array}$ & $\begin{array}{r}\text { \% Error in } \\
\text { mean }\left(f_{3}\right)\end{array}$ & $\begin{array}{l}\text { Estimate of } \\
P_{\text {failure }}\end{array}$ \\
\hline Continuum Solution & $n / a$ & $\mathrm{n} / \mathrm{a}$ & $\mathrm{n} / \mathrm{a}$ & $0.00 \%$ & $2.75 \%$ \\
\hline RSM w/mesh size & 25 & 6 & 59 & $0.01 \%$ & $2.69 \%$ \\
\hline RSM on fine mesh & 13 & 13 & 94 & $0.27 \%$ & $1.61 \%$ \\
\hline $\begin{array}{l}\text { Direct } \mathrm{MC} \text { on } \\
\text { nominal mesh }\end{array}$ & 100,000 & 0 & 90,000 (est) & $1.09 \%$ & $0.23 \%$ \\
\hline
\end{tabular}

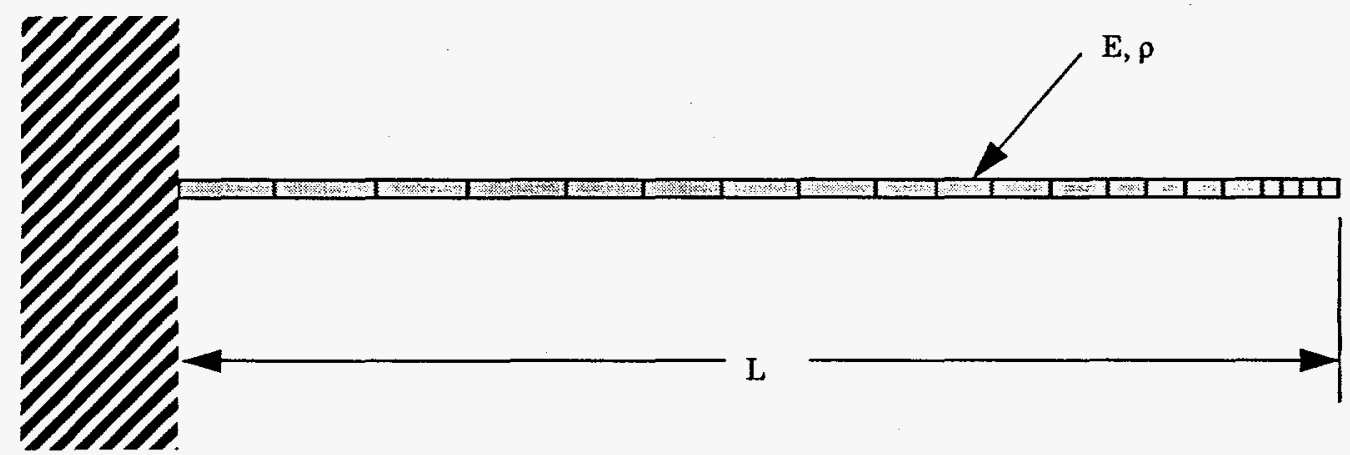

Figure 1: 1-D Prismatic Bar Model with Nominal Mesh 


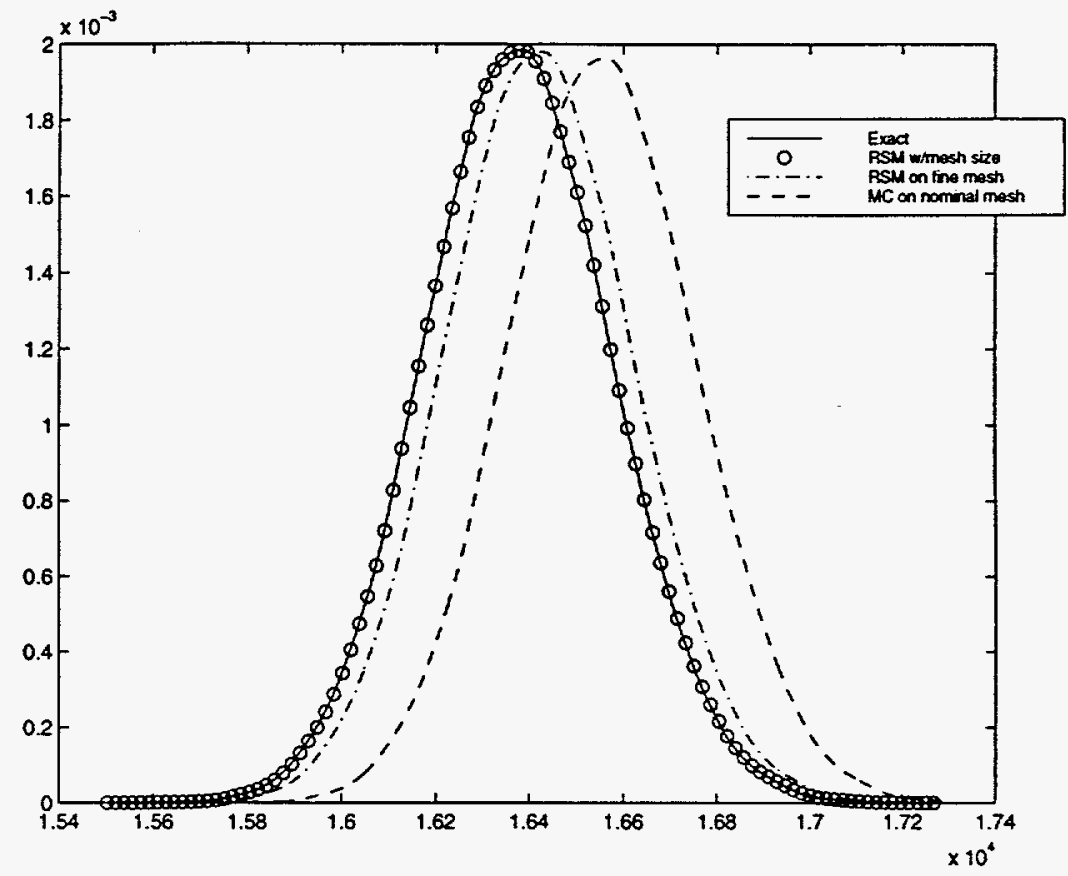

Figure 2: Results from Nondeterministic Analysis of 1-D Bar

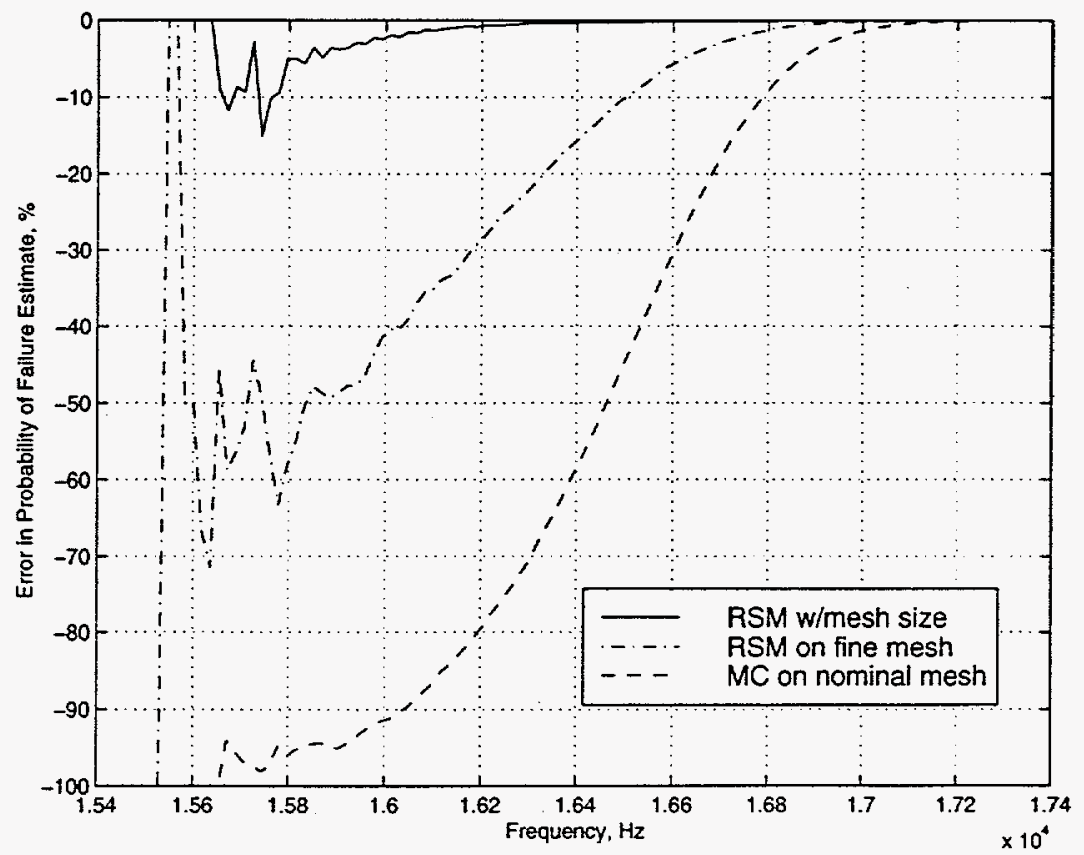

Figure 3: Error in $P_{\text {failure }}$ as a function of Failure Threshold 


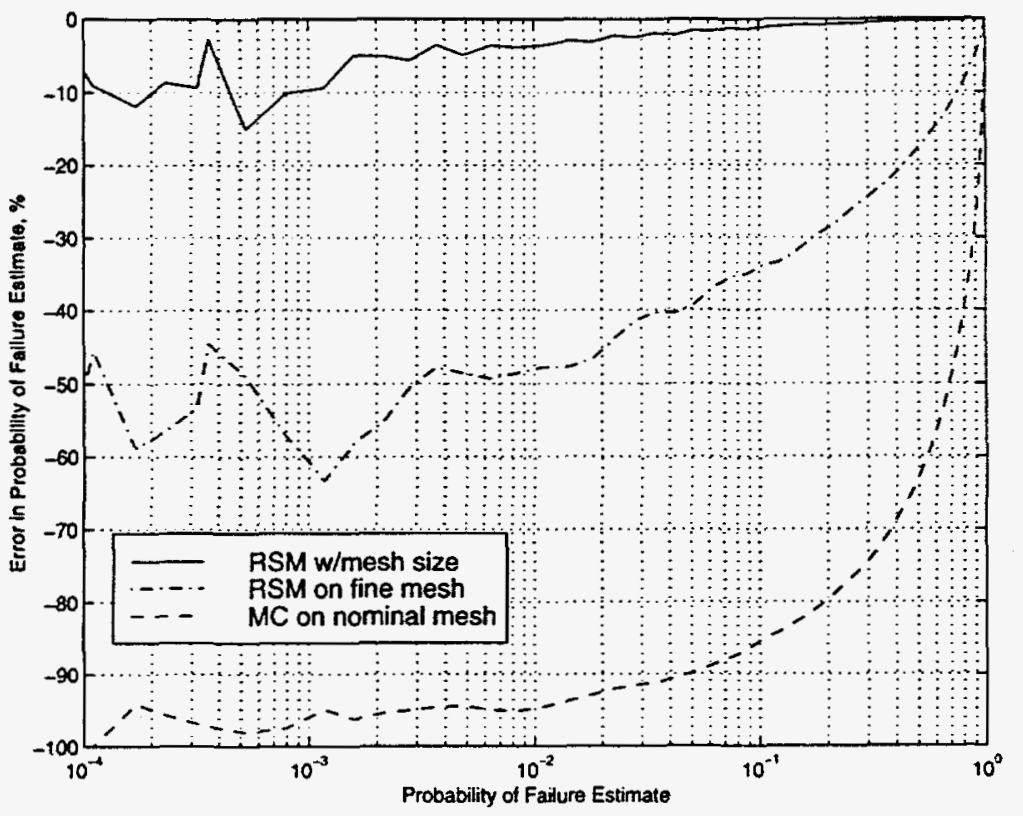

Figure 4: Error in $P_{\text {failure }}$ as a function of $P_{\text {failure }}$ 